Artículos / Articles

\title{
Uneasy riders: contradictorias lógicas disciplinarias para una posición laboral imposible
}

\author{
Uneasy riders: contradictory disciplinary logics for an \\ impossible job position
}

\author{
Juan Carlos Revilla \\ Departamento de Antropología Social y Psicología Social, UCM \\ Instituto TRANSOC, UCM \\ jcrevilla@cps.ucm.es \\ Víctor Blázquez Martín \\ Departamento de Historia, Teorías y Geografía Políticas, UCM \\ vbmartin@ucm.es \\ Recibido / Received: 29/07/2020 \\ Aceptado / Accepted: 04/11/2020

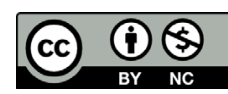

\section{RESUMEN}

En esta investigación analizamos las lógicas disciplinariasy formas de control organizacional existentes en el reparto de comida a domicilio bajo la economía de plataforma. La situación laboral de los repartidores o "riders" supone un cambio significativo en las formas en que se desarrolla este proceso y en las lógicas organizacionales que definen su posición. Estos trabajadores están sometidos a distintos mecanismos disciplinarios que contribuyen a asegurar su buen desempeño, tanto mediante el control algorítmico, como a través de una gestión de la subjetividad que discursivamente enfatiza la flexibilidad y autonomía. Este es precisamente el factor disruptivo en el ámbito laboral que estas plataformas reclaman. Sin embargo, el carácter contradictorio de esas lógicas disciplinarias dibuja una situación que parece imposible, o al menos bastante difícil, de vivir, lo que les convierte en trabajadores incómodos (uneasy riders).

Palabras clave: lógicas disciplinarias, control organizacional, control algorítmico, gestión de la subjetividad, repartidores.

\begin{abstract}
In this research we analyze the disciplinary logics and existing forms of organizational control in food delivery under the platform economy. The working situation of couriers or "riders" represents a significant change in the ways this process is carried out and in the organizational logics that define their position. These workers are subjected to different disciplinary mechanisms to help ensure their good performance, through both algorithmic control and subjectivity management that discursively emphasizes flexibility and autonomy. This is precisely the disruptive factor in the labor environment that these platforms claim. However, the contradictory character of these disciplinary logics draws a situation that seems impossible, or at least quite difficult, to live, which turn them into uneasy riders.
\end{abstract}

Keywords: disciplinary logics, organizational control, algorithmic control, subjectivity management, riders. 


\section{INTRODUCCIÓN}

No cabe duda de que la capacidad de los medios tecnológicos para el control social es objeto de interés e incluso preocupación, pues tienen cada vez mayor capacidad de recoger y procesar los múltiples datos que se generan a partir de la actividad social. Estos recursos tecnológicos han llegado también al mundo del trabajo en forma de mecanismos de control cada vez más sofisticados para asegurar el desempeño requerido en las diferentes posiciones laborales. Paradójicamente, esta intensificación de las formas de control convive con discursos organizacionales que enfatizan los beneficios de la autonomía o autogestión del trabajador (ver Boltanski y Chiapello, 1999), lo que habría de significar necesariamente la utilización de mecanismos de control más discretos (Fleming y Sewell, 2002) y a los que es más dificil resistirse.

Las nuevas formas de economía de plataforma, que involucran ya a alrededor del $10 \%$ de la población trabajadora (Pesole, Urzí, Fernández-Macias, Biagi y González, 2018), estarían aportando nuevas formas de control atravesadas por los desarrollos tecnológicos en los que se basan estos procesos de trabajo. Este trabajo plantea el análisis de las lógicas disciplinarias y formas de control existentes en el trabajo de reparto de comida preparada a domicilio. Estos trabajadores (repartidores o "riders") están sometidos a distintos mecanismos disciplinarios que contribuyen a que la empresa se asegure su buen desempeño, tanto mediante el control algorítmico, como a través de la dirección de la empresa y de una serie de discursos organizacionales que enfatizan la flexibilidad y autonomía que tiene el trabajador para decidir cuándo y cuánto desea trabajar como tal repartidor.

\section{Control y disciplinamiento en las organizaciones}

La teoría del proceso de trabajo y los estudios críticos del management han identificado, las formas clásicas de control organizacional, asi como las lógicas disciplinarias subyacentes (ver Revilla y Tovar, 2011). La lógica panóptica, basada en la vigilancia, ha evolucionado de formas de control simple (Edwards, 1979) a otras mediadas tecnológicamente (control tecnopanóptico; Revilla y Tovar, 2011). La lógica formalizadora, basada en la estandarización de procesos tecnológicos o administrativos, se ha visto expulsada de muchas formas de trabajo más dificiles de estandarizar en el actual régimen postfordista, pero permanece también ayudada por las nuevas tecnologías que simplifican los procesos productivos.

La investigación en el campo también ha identificado nuevas lógicas disciplinarias y formas de control organizacional, que tienden todas ellas a ocultar la relación de dependencia directa del trabajador respecto de la autoridad organizacional. Así, la lógica productivista tiende a permitir la autoorganización del trabajador en el establecimiento de unos medios para conseguir los fines establecidos por la organización, dando al trabajador una mayor sensación de autonomía. Esta lógica incluye el control clientelar, es decir, la necesidad de satisfacer al cliente como elemento inherente a la correcta realización del trabajo (Boltanski y Chiapello, 1999; Dubar, 2002). La lógica relacional, mediante el control concertivo (Barker, 1993), hace depender el desempeño de la responsabilidad compartida en el equipo de trabajo.

El control a través de la gestión de la cultura organizacional (Kunda, 1992; Wilmott, 1993), que Frenkel, Korczynski, Donoghue y Shire, K. (1995) denominaron control "infonormativo", estaría vinculado con estas nuevas formas de control, en la medida en que la dependencia de una autoridad se sustituye por el intento persuasivo de que el trabajador interiorice determinadas normas y valores propios de la cultura de la organización, como forma de asegurar un modo de hacer en contextos presididos por la autonomía en la toma de decisiones. Este tipo de control sería igualmente más discreto o menos evidente y se 
relaciona con culturas de alto rendimiento o excelencia y puestos de trabajo que precisan de flexibilidad funcional y alta autonomía (Alvesson y Willmott, 2002; Fleming y Sewell, 2002).

Por último, algunos autores (Alonso y Fernández, 2009; Revilla y Tovar, 2011) están señalando que la flexibilidad y la precariedad pueden convertirse en una emergente lógica disciplinaria fluida, basada en la indefensión de los trabajadores ante unas condiciones laborales siempre cambiantes en aras de la adaptación a un mercado cambiante.

Algunas nuevas formas de trabajo pueden poner a prueba estas clasificaciones y mostrar la emergencia de nuevos mecanismos, o la profundización en alguno de los existentes. Además, el análisis de posiciones laborales específicas (ver por ejemplo Sewell y Wilkinson, 1992) puede permitir apreciar la convivencia, en el mismo contexto laboral, de lógicas disciplinarias distintas que pueden retroalimentarse y aumentar su capacidad de éxito en el objetivo del control de los trabajadores y el logro de los objetivos de la organización.

\section{Economía de plataformas y control organizacional}

En este contexto, la economía de plataformas (gig economy) o el trabajo de multitudes (crowd work) es un campo de estudio interesante, en la medida en que a la utilización de herramientas tecnológicas (muchas basadas en algoritmos y el aprendizaje de las máquinas) se une una intensa retórica basada en la libertad, autonomía y flexibilidad de los colaboradorestrabajadores. Tomamos aquí la definición de Huws, Spencer, Syrdal y Holts (2017) de trabajo de multitudes como el trabajo pagado a través de una plataforma online (ver también Huws, 2014). Dentro de este, podemos diferenciar, siguiendo a Valenduc y Vendramin (2017), entre el trabajo de microtareas virtuales (ej.: Amazon Mechanical Turk), el de tareas profesionales (ej.: plataformas para traducciones) y el de servicios y tareas materiales (ej.: conductores y "riders"). Aunque hay diferencias importantes, todos estos tipos de trabajo comparten la sustitución de la relación asalariada tradicional por una relación, al menos, triangular, donde la plataforma media entre cliente y el trabajador. Esta relación triangular es compartida por otras iniciativas de la llamada economía colaborativa, pero la economía de plataforma se aleja en otros muchos aspectos de cualquier intención comunitaria o emancipatoria que sí está presente en aquella (ver Alonso, 2017; Piñeiro, Surinach y Casadevante, 2017).

De hecho, lo que consigue la economía de plataforma con esta relación a tres es constituir una fuerza de trabajo flexible y de bajo coste, disponible bajo demanda, que provee a la plataforma de sus servicios como trabajadores independientes. Así, aparte de minimizar los costes del trabajo, la plataforma no genera ningún tipo de compromiso con los trabajadores, sobre quienes descansan todos los riesgos asociados con el ejercicio de sus tareas: materiales para el trabajo, accidentes, enfermedades, etc. (De Stefano, 2016; Tassinari y Maccarrone, 2020). Con ello, se vuelca el riesgo empresarial sobre los repartidores.

En estos procesos productivos está presente normalmente algún tipo de algoritmo, ajustado a las características del trabajo que se realiza. Esto ha llevado a acuñar el término de gestión algorítmica (Lee, Kusbit, Metsky y Dabbish, 2015; Schildt, 2017), para referirse a la sustitución, al menos parcial, de la gestión de personas por desarrollos informáticos que usan grandes cantidades de datos para organizar el trabajo de la manera más eficiente y que son capaces de "aprender" en función de los cambios que se producen en el entorno. Esto abre debates sobre las características de este tipo de gestión y sobre las nuevas formas de control sobre los trabajadores que posibilita la gestión algorítmica, lo que ha dado también lugar a hablar de control algorítmico (Veen, Barratt y Goods, 2020).

La emergente literatura sobre los repartidores ha puesto de manifiesto las características de la gestión algorítmica y las distintas formas de control a las que están sometidos. El algoritmo reemplaza en gran medida a la gestión tradicional, de forma que la aplicación a la que se conectan los repartidores se convierte en el punto real de producción, pues por ella pasan todo el proceso de trabajo. Cameron (2020) señala cinco tipos de algoritmos que 
coordinan el ciclo laboral: a) asignación de repartidor al cliente; b) instrucciones para realizar el trabajo (ruta, tiempos, etc.); c) ajuste de precio del pedido; d) oferta de bonos; e) evaluación del desempeño. Por ello, con el desarrollo completo de los algoritmos, la dirección de la empresa queda completamente oculta bajo el funcionamiento de estos, que van ajustando sus resultados a la situación a partir de los datos acumulados por experiencias previas.

El control de los trabajadores pasa también, en gran medida, por el algoritmo. La dirección de la empresa supervisa todo el proceso y actúa cuando lo considera conveniente, lo que configura una forma de control multifacética o híbrida (Veen et al., 2020). Los algoritmos vigilan a los repartidores constantemente (lógica panóptica) mediante su interacción con la aplicación y las herramientas de geolocalización. Pero, al tiempo, estandarizan técnicamente el trabajo (lógica formalizadora, control tecnológico), de una forma que algunos autores denominan taylorismo digital (Cherry, 2016) o administración científica 2.0 (Schildt, 2017). Se encargan también de la evaluación del trabajo, a través de métricas de desempeño o de calificaciones de los clientes (Veen et al., 2020; Huber y Scheytt, 2018), lo que estaría más cerca de la lógica productivista.

Por otro lado, muchos autores (Huber y Schwytt, 2018; Veen et al., 2020, Tassinari y Maccarrone, 2020; Cameron, 2020) señalan la opacidad en la creación y efectos de los algoritmos en el entorno laboral, al tiempo que enfatizan también los constantes cambios que sufren, siempre en aras de una mejor efectividad y ajuste al mercado, lo que tiene efectos de indeterminación sobre los trabajadores (lógica fluida).

Por último, se ha insistido en los esfuerzos de las organizaciones por difundir unos valores entre los trabajadores (Veen et al., 2020) en términos de una cultura de orientación hacia el cliente y de flexibilidad, que valore la autonomía de la que aparentemente disfrutan los repartidores.

A partir de esta revisión, en el presente trabajo analizamos el carácter contradictorio de las lógicas disciplinarias a las que están sometidos estos trabajadores, como forma de incidir sobre la situación imposible, o poco sostenible, a la que les conduce. Para ello, se reflexionará sobre las lógicas disciplinarias de los repartidores a partir de una investigación propia, que incluirá las formas de gestión de su subjetividad a las que están sometidos, pues ambos elementos forman parte indisociable de este contexto laboral.

\section{METOdOLOGÍA}

El presente trabajo se basa en diversas fuentes empíricas para el análisis de las lógicas disciplinarias y las formas de control que utiliza la compañía para asegurarse el buen funcionamiento de la producción y para el análisis de las formas de gestión de la subjetividad que pone en marcha. Con ello se pretende mostrar el carácter complejo, multifacético, incluso contradictorio, de este tipo de trabajo.

Para las lógicas disciplinarias y las formas de control, se analizan materiales obtenidos mediante técnicas etnográficas (Hammersley y Atkinson, 1994; Denzin, 1997) de observación participante, aplicadas a la experiencia de un varón ${ }^{1}$ que trabajó en Madrid en el sector de la comida a domicilio, primero como repartidor para dos de las plataformas de reparto de comida a domicilio más conocidas en España y después como gestor logístico.

Como repartidor, la experiencia se produjo entre agosto de 2015 y febrero de 2016, cuando estas organizaciones empezaban a implantar su actividad en la ciudad. Inicialmente, la relación con la organización era mucho más directa y fluida. Al inicio era común una organización semi-formal de 'jefes de equipo', a la que se tuvo acceso. Los escenarios informales de contacto eran numerosos: centroides, espacios virtuales de comunicación entre repartidores y jefes de equipo, actividades comerciales organizadas por la empresa en

1 Por razones de anonimato se evita identificar las plataformas en la que trabajó el sujeto. Baste decir que se trata de plataformas líderes en el sector en nuestro país. 
las que participan repartidores, así como otros de carácter no laboral. Entre las actividades 'formales' se situarían los canales establecidos para las comunicaciones plataformarepartidor, las reuniones periódicas y extraordinarias de jefes de equipo y, en algunos casos, actividades lúdicas establecidas entre las plataformas y los repartidores.

Como gestor logístico, la experiencia tuvo lugar entre febrero de 2016 y noviembre de 2017. En ella existieron distintos tipos de interacción formal de diversa amplitud en la organización: actividades de coordinación general, nacionales e internacionales; actividades de coordinación interdepartamental e intradepartamental, nacionales e internacionales; y reuniones personales de coordinación con diversas posiciones en la estructura organizativa. Las actividades informales incluyen: conversaciones durante el trabajo o en sus tiempos muertos, individual y grupalmente; y en los espacios fuera del ámbito de trabajo, actividades como comidas e "ir a tomar algo" con algún trabajador particular, o comidas, cenas, fiestas y convivencias organizadas por la compañía.

Durante ese tiempo se acumuló un amplio conocimiento del funcionamiento de las plataformas, que se dio forma en numerosas notas de campo. Esto supuso también un trabajo de elaboración selectiva y retrospectiva de experiencias pasadas, lo que lo acerca a la experiencia autoetnográfica (Ellis, Adams y Bochner, 2011), sobre todo a la de corte analítico (Anderson, 2006). Esta ha sido tratada de un modo similar al que plantea Bourdieu (2006), induciendo una reflexividad y reconversión de la mirada que hace posible la "recuperación de la relación práctica con el mundo" y el "acceso a la verdad de las prácticas" (Bourdieu, p. 90).

El proceso de sistematización de esa experiencia se reforzó con la realización de una entrevista en profundidad ( 25 de febrero de 2020) como técnica vehicular para su ordenación y desvelamiento entre el etnógrafo y un investigador de amplia experiencia en el campo.

En este caso, el uso de herramientas (auto)etnográficas ayuda a conocer el diseño, implementación y formas de interacción entre los trabajadores y el algoritmo, ante la dificultad de observar su funcionamiento. La experiencia en una posición operativa puede aportar una perspectiva de cómo se automatizan algunas tareas mediante el uso de 'inteligencia artificial', que pasarán a ser competencia del algoritmo.

Para el análisis de la gestión de la subjetividad, se han trabajado materiales significativos de la relación de la plataforma con sus repartidores y que han servido para reconstruir las distintas formas de interpelación de la compañía. En primer lugar, la declaración de principios de la plataforma y de la relación entre repartidores y comunidad (acceso en junio de 2020). En segundo lugar, las comunicaciones online entre la dirección logística de la empresa y los repartidores (entre junio de 2016 y junio de 2017), recogidas en el "Dossier de pruebas" que presentaron la Plataforma RidersXDerechos y el sindicato Intersindical Alternativa de Catalunya, como denuncia a una de estas empresas ante la Inspección de Trabajo de Barcelona en 2017. En tercer lugar, las historias de repartidores concretos que aparecen en la web de la plataforma (acceso junio de 2020) y que nos muestran ejemplos virtuosos de este tipo de trabajadores, ajustados a las demandas y pretensiones de la compañía. Por último, se analiza el contenido, no los datos, de una encuesta de satisfacción de los repartidores (diciembre 2017) en su relación con la compañía, pues genera un marco definido para la relación entre las partes.

El trabajo etnográfico ha confirmado la masculinización y juvenilización importante de la profesión de repartidor en la plataforma analizada. Esto coincide con investigación reciente (ver Pesole et al., 2018), que ha mostrado que la media de edad de los trabajadores de las plataformas es inferior a la de la población general. Estos mismos autores han evidenciado también la menor presencia de mujeres en este rubro, desigualdad mayor cuanto más intensa es la dedicación a estas tareas. Esta desigualdad tendrá presencia en el análisis de la gestión de las subjetividades, en el que se apreciará el sesgo masculino de las subjetividades propuestas. 
El análisis realizado se caracteriza como análisis crítico de discurso, orientado desde la Teoría del Proceso de Trabajo y los planteamientos foucaultianos sobre el disciplinamiento en el entorno laboral.

\section{RESULTADOS}

\section{Control algoritmico}

El aumento en la capacidad de control sobre los procesos laborales de las herramientas algorítmicas parece ser una de las notas distintivas de la economía de plataforma. La novedad en las plataformas digitales parece desafiar no sólo la agencia individual y colectiva de los trabajadores, sino también las metodologías y tipologías que las ciencias sociales han utilizado para el análisis de las relaciones laborales.

Sus características postfordistas y neotayloristas pueden crear la ilusión de que la gerencia algorítmica ha copado totalmente el ámbito de las formas de control y disciplina de la fuerza de trabajo. Con ello, se pueden destacar al menos dos dimensiones de las nuevas plataformas digitales: su capacidad de transformación sobre el contexto socioeconómico resultante de la última crisis financiera, lo que en muchas ocasiones ha sido llamado su capacidad disruptiva; y las contradicciones inherentes a estas formas de control organizacional debido a las distintas lógicas disciplinarias que pone en práctica.

Sin embargo, en este análisis veremos que la mayor parte de los mecanismos que se ponen en marcha con el funcionamiento de los algoritmos se pueden encuadrar en la investigación existente. Junto al control algorítmico, que ya parece implicar distintas lógicas de control, aparecen otras formas de control de la fuerza productiva, en línea con la hibridación de las formas de control del proceso de trabajo (Veen et al., 2020).

El elemento central para la definición de la gestión del proceso de trabajo en el reparto de comida a domicilio y, por tanto, de las llamadas plataformas digitales en que se imbrican, es la utilización de tecnologías basadas en el cálculo operativo. A partir de ingentes cantidades de información, mediante algoritmos, han creado sistemas capaces de organizar a gran escala y aumentar la eficiencia de ciertos sectores productivos. En este caso, el algoritmo es el resultado de un conglomerado computacional jerárquico capaz de coordinar y controlar, mediante su conversión en datos operativos, todos los componentes implicados en la red de reparto. Desde el punto de vista del proceso de trabajo, existen distintos algoritmos para diferentes funciones: a) asignación de pedidos a los repartidores; b) producción y comunicación on time de las indicaciones necesarias en todas las fases del proceso (aceptación del pedido, ruta al restaurante, llegada al restaurante, contenido del pedido, indicación de pedido preparado, ruta al cliente, entrega de pedido); c) ajuste dinámico del precio de cada reparto; d) evaluación del rendimiento de cada repartidor en función de distintos indicadores o métricas. En el análisis veremos cómo cada uno de ellos tiene implicaciones distintas de cara al proceso de trabajo y al control sobre los trabajadores, lo que puede estar detrás de ese carácter contradictorio que observamos en este tipo de organización del trabajo.

\section{Lógica panóptica}

El trabajo de los repartidores comparte con otras formas de trabajo la vigilancia mediante los sistemas de posicionamiento geográfico (GPS), que están disponibles en los dispositivos móviles personales de los riders (hardware) y que son controlados a través de la aplicación (app) de la compañía (software). Esta aplicación tiene que estar instalada y ser utilizada por todos los repartidores para poder llevar a cabo su trabajo, por lo que aparece como el punto de producción principal (Veen et al., 2020). La empresa, y sus algoritmos, conocen en tiempo real 
la ubicación de sus trabajadores, lo que permite controlar constantemente sus movimientos y reaccionar ante posibles incumplimientos. Así, los repartidores reciben mensajes cuando se desvían o incumplen alguna de las directrices que rigen el proceso de entrega de pedidos, a los que deben responder de forma rápida interactuando con la aplicación. Del mismo modo, en esos mismos casos los gestores reciben mensajes de alarma estandarizados a partir de la información que se recoge del proceso. Con ello, se reduce significativamente la supervisión directa, por la interacción con una aplicación que media en el control del proceso de trabajo.

Por tanto, estaríamos en presencia de un control tecnopanóptico (Revilla y Tovar, 2011), el mismo al que están sometidos todo tipo de repartidores desde el desarrollo de los sistemas GPS y los teléfonos inteligentes. En ese sentido, estos sistemas de geoposicionamiento permiten ampliar espacialmente el control organizacional de manera inusitada, haciendo posible este tipo de lógica disciplinaria en procesos de trabajo dificilmente reducibles a los espacios laborales convencionales (fábrica u oficina). Su contexto, por tanto, no estaría definido por un espacio cerrado, sino por un espacio extensivo como campo de aplicación. Si bien no hay un centro de trabajo único donde se ejerza la vigilancia a través de la disposición del espacio, el control del proceso productivo por geolocalización convierte a la ciudad entera en un eficiente panóptico.

\section{Lógica formalizadora}

El control sobre el proceso laboral se ejerce también a través de una serie de pautas institucionalizadas que prescriben distintos tipos de acciones y comportamientos $-y$ se ejerce vigilancia sobre su ajuste y cumplimiento- mientras se espera la llegada de pedidos, cuando se llega al restaurante y cuando se llega a casa del cliente. Buena parte de estas acciones se producen en la interacción entre el trabajador y la aplicación (a través del smartphone). Ésta es la forma por la que deja constancia de que se van completando las fases del pedido, al tiempo que permite el control logístico de los algoritmos desarrollados por la compañía. Así, solo se puede pulsar el botón "ya he llegado" a pocos metros del restaurante y solo cuando se hace correctamente aparecen los ítems del pedido que se va a transportar al cliente.

Por tanto, la aplicación es la mediadora formal en las interacciones con la compañía, clientes, otros trabajadores y consumidores, siendo elemento fundamental en el orden gerencial que rige el proceso laboral. La centralidad funcional y operativa de la app remitiría a una cierta racionalidad instrumentalizada en la organización de la producción. Su mecánica establece una serie de acciones estandarizadas cuya finalidad es aumentar la eficiencia del proceso. La lógica que guía el proceso está diseñada para evitar en la mayor medida posible cualquier tipo de interferencia que ralentice el proceso de reparto y produzca ineficiencias. De esta manera, se establecen formas y tiempos de relación con el espacio, controlando las interacciones que en él se producen.

Pero la clave de esta estandarización de procesos reside en los algoritmos detrás de la aplicación, que establecen la forma y los ritmos de ejecución del trabajo. El algoritmo trabaja sobre un ingente flujo acumulativo de información -big data- proveniente de todos los casos de reparto anteriores, que se acumulan en una base de datos. La comunicación del sistema con el repartidor se expresa, pues, en el doble flujo de recepción de información y generación de órdenes a los elementos de la red de reparto. Por tanto, la primacía jerárquica en la actividad del trabajador corresponde a la app (y a los algoritmos que están detrás). Solo cuando se produce algún tipo de incidencia no recogida en las opciones resolubles por el algoritmo aparece la interacción del repartidor con otro trabajador de la plataforma que le da soporte. 
Estaríamos hablando, entonces, de un control tecnológico intensivo con una infraestructura de comunicaciones altamente centralizada (Bodo et al., 2017) que une y coordina todas las interacciones que se producen en su "ecosistema digital" (Veen et al., 2020 , p. 390). Este control viene dirigido por el segundo componente del algoritmo señalado más arriba, que se encarga también de la vigilancia tecnopanóptica de los repartidores. Este algoritmo necesita de una mano de obra extremadamente dócil y disciplinada que haga en cada momento lo que es preciso de la manera más eficiente y productiva (en tiempos y movimientos). Esto nos sitúa ante la impersonalidad de una interacción mecanizada a través de la app, llevada a su paroxismo post -o hiper- moderno en las plataformas digitales. Toda la elaboración inherente al proceso de trabajo, expresada principalmente en los sistemas de comprobación del cumplimiento de las órdenes emitidas por la aplicación, parece remitir a una nueva forma de deshumanización neotaylorista de las relaciones laborales en la que el trabajador se convierte en poco más que un apéndice de la máquina. De hecho, la robotización y automatización del reparto que se han anunciado en algún momento como perspectiva de futuro parece confirmar de algún modo este extremo. En esta lógica disciplinaria parece estar implícita la idea de que la respuesta más productiva es la que procede de la máquina. Podríamos hablar, por tanto, de una praxis institucionalizadora del espacio y el tiempo, y con ello de una nueva forma de taylorización. Esto contrasta vivamente con el discurso de la autonomía que se sostiene desde la compañía, como desarrollamos más adelante, en su intento de justificar la ausencia de relación laboral asalariada entre repartidores y empresa.

Por tanto, la plataforma necesita repartidores que se comporten casi como autómatas, lo que casa mal con su estatuto de trabajadores autónomos, pero también con la inherente indeterminación del trabajo en toda relación laboral (Edwards, 1990). De hecho, la compañía ha puesto en marcha mecanismos que refuercen (y convenzan a legisladores y jueces de) la autonomía de los trabajadores, como la posibilidad de rechazar un pedido previamente asignado. Pero, al tiempo, se han desarrollado mecanismos para conseguir la aquiescencia de los riders con el ánimo de mantener la tan necesaria eficiencia de los procedimientos. La compañía clasifica a sus repartidores en función de su rendimiento, lo que incluye los tiempos medios de entrega, el cumplimiento de las normas, la tasa de rechazo de pedidos, etc. Por tanto, rechazar un pedido, elemento de autonomía como trabajador, tiene consecuencias que se manifiestan de una manera oscura, pero palpable, en peores posibilidades de acceso al reparto: los repartidores con mejores evaluaciones son los primeros en elegir franjas horarias de reparto, por ejemplo.

A pesar de esto, el procedimiento de trabajo podría hacerse más llevadero por la tendencia a una cierta gamificación del reparto. Sería visto entonces como un juego en el que el repartidor trata de conseguir más ingresos a partir de las reglas que establece el algoritmo. Entendemos que estos incentivos operan en la subjetividad de modo similar a como lo planteaba Burawoy (1979), y tal como lo hacen en la actualidad otro tipo de juegos virtuales. A esto se añade la realización de actividades básicas que pueden aparecer como una afición o un disfrute, como montar en bici o en moto, y sin la presencia permanente de un jefe que haría menos estimulante la participación en el juego. Así, sobre la base de una cierta tendencia a familiarizarnos con las herramientas y acostumbrarnos a ser observados (Huber y Schwytt, 2018), puede ser expresada una sensación de autonomía y de mayor libertad en respuesta al control algorítmico, comparado con el control ejercido directamente por un jefe (Cameron, 2020).

\section{Lógica productivista}

Esta lógica también está presente en esta relación laboral, también de una manera contradictoria. Se fundamenta en la autorregulación a que estaría sometido el trabajador para obtener unos determinados resultados productivos, sea en términos de logro de 
objetivos, satisfacción del cliente o adaptación productiva al mercado. La contradicción se sitúa precisamente en la aparición de esta lógica disciplinaria en un contexto en el que la estandarización de procesos es tan intensiva, tal como hemos señalado. En el control habitual por resultados (Drucker, 1979) es la organización la que fija los objetivos y el trabajador tiene la autonomía para alcanzarlos de la manera que estime conveniente. En el caso de los repartidores, no existe apenas autonomía para la autoorganización del trabajo más allá de elegir la ruta en los desplazamientos. Pero esto estará siempre condicionado por la compulsión de realizarlo en el menor tiempo posible, tal como es indicado por la aplicación. Pero al tiempo existiría una aparente autonomía para fijar los objetivos de trabajo, en la medida en que el rider puede elegir los tiempos en los que trabajar y el número de horas de reparto. Sin embargo, la compañía exige trabajar un número mínimo de horas y que un determinado porcentaje de ellas se realicen en los periodos de mayor demanda en los fines de semana.

En este sentido podríamos hablar de un tipo de control mercantil (Revilla y Tovar, 2011), en la medida en que la regulación del trabajo pasa por la adaptación a una demanda difícil de anticipar, aunque a esto se consagran precisamente las funcionalidades de los algoritmos. Al hacer partícipes a los repartidores de las adaptaciones a la demanda, la plataforma 'externaliza' sus fluctuaciones dejando que recaiga en buena medida sobre ellos. Así, quedan expuestos a una determinación directa del mercado sobre su trabajo. Aunque es la organización la que realiza la previsión sobre la demanda, y no los repartidores, si los cálculos fallan, en el mejor de los casos las consecuencias podrán ser en parte compartidas, pero siempre afectarán a la organización del trabajo de los repartidores.

Lógicamente, esta forma de trabajo también tiene elementos de control clientelar por la relación que se establece con los clientes finales (ver Dubar, 2002; Boltanski y Chiapello, 1999), pero también con los restaurantes asociados. Si bien la interacción con ambos actores es muy limitada (recepción-entrega de productos), esto no elimina el escrutinio de la compañía. Por un lado, se realizan indicaciones de cómo han de ser las interacciones con estos actores, como veremos más adelante. Por otro, se recogen indicadores acerca de esa relación con el cliente. En algunas plataformas el cliente evalúa al repartidor, lo que es una forma clara de medir su satisfacción, algo típico del control clientelar. Pero en todos los casos se tienen en cuenta otros indicadores que implican la capacidad de control del cliente sobre el proceso laboral, como las quejas o reclamaciones, o la interacción del repartidor con la app que hace posible el seguimiento del pedido.

\section{Lógica fluida}

En la lógica fluida se expresan con agudeza algunas de las contradicciones a las que ya hemos aludido. Esta lógica, típicamente postfordista, se fundamenta en la ausencia de unos principios de organización y recompensa estables y en el disciplinamiento a través de la incertidumbre que esto genera, ya sea en términos de flexibilidad funcional o numérica (Revilla y Tovar, 2011), y se justifica en la necesidad de adaptarse a los cambios del entorno.

A este respecto, la flexibilidad funcional pasa por las operaciones de los algoritmos, tanto en la asignación de pedidos, como en la determinación del precio del reparto o en la evaluación del desempeño. Es muy notoria la aparente arbitrariedad y oscuridad con que el algoritmo realiza el proceso de asignación de pedidos, sobre los criterios por los que algunos pedidos tienen un precio mayor ("surges") o sobre los que determinan una mejor o peor evaluación del repartidor, tal como han señalado Veen et al. (2020). Esto genera una asimetría en la información de la que disponen las dos partes implicadas en el proceso para beneficio de la compañía (Veen et al., 2020; Tassinari y Maccarrone, 2020; 
Bodo et al., 2017; Cameron, 2020). Por ejemplo, el repartidor no conoce la dirección del cliente en el momento de aceptar el pedido, sino cuando lo ha recogido en el restaurante, lo que asegura la aquiescencia del trabajador y limita sus posibilidades de actuación autónoma. Por otro lado, los aumentos de precio de algunos pedidos están relacionados con el hecho de que se consideran más difíciles de asignar por el lugar o el momento en que se producen, de forma que es una manera de fomentar el interés en realizarlo, es decir, de asegurar esa misma aquiescencia.

Además, la naturaleza adaptativa y cambiante de los propios algoritmos contribuye aún más a esta continua incertidumbre inmovilizadora que implica la lógica fluida. El algoritmo "aprende", de forma que modifica el resultado de su funcionamiento en función de los datos recopilados hasta ese momento. Esto, entre los repartidores, puede ser percibido como un cambio de criterio o de política de la compañía. De hecho, los desarrolladores de los algoritmos modifican los parámetros que establecen los márgenes dentro de los que se producirá su funcionamiento, a partir de las indicaciones que la dirección de la compañía pueda establecer. Incluso en algunos casos se ha producido la implementación de un nuevo algoritmo para permitir, según la empresa, un mejor funcionamiento del proceso de trabajo y una mejora de las condiciones para los repartidores. Con ello, se produce de facto una continua renovación de la organización del trabajo y de sus normas y parámetros de evaluación, lo que refuerza igualmente la sensación de indefensión propia de la lógica fluida, amplificada por la mencionada opacidad en el funcionamiento de los algoritmos.

Con ello, el trabajador está continuamente expuesto a una organización inestable y fluida en su trabajo, que contradice significativamente la lógica formalizadora previamente abordada. Estaríamos, pues, ante un proceso de desinstitucionalización (Berger y Luckman, 1986), caracterizado por la ausencia de una norma clara y permanente. Pero, quizá, este proceso de institucionalización y desinstitucionalización se mueva en dos niveles distintos, intencionalmente divididos para sostener la lógica fluida: se reclama la flexibilidad del trabajador, mientras se institucionaliza la soberanía del algoritmo como elemento performativo en la gestión del trabajo.

En cuanto a la dimensión numérica de la lógica fluida, nos encontraríamos ante la continua posibilidad de expulsión de la organización si se producen incumplimientos graves por parte del trabajador. La perenne amenaza de perder el empleo ejercería una significativa presión para aceptar la propia condición ‘fluida' de la situación. La amenaza aumenta su intensidad, y, por tanto, la soberanía del algoritmo, en la medida en que el proceso de despido queda reducido a una mera 'desconexión'. Pero la oscuridad en el control algorítmico, que le hace aparecer como una 'caja negra' (Cant, 2020), también parece implicar una significativa arbitrariedad en el proceso de eventual desconexión. La compañía cuenta con la ventaja de poder incorporar nuevos trabajadores en cualquier momento, y de hecho siempre está abierta la posibilidad de incorporación a través de la web, a pesar de la elevada tasa de abandono que existe entre los repartidores.

Pero en este caso, y como resultado también de su estatus no asalariado, se produce una paradoja. Lógicamente, a los riders no les interesa que el número de repartidores sea muy grande, pues esto reduce los recursos que se pueden obtener por el trabajo, puesto que resultará en menos pedidos e ingresos por hora de trabajo. Por el contrario, la empresa sí podría estar interesada en disponer del mayor número posible de repartidores, sobre todo ahora que los algoritmos son capaces de manejar grandes cantidades de datos en tiempo real. Con ello, conseguiría presionar a los riders para la aceptación de las condiciones de trabajo y la aceptación acrítica de las normas que lo regulan. Por supuesto, el límite a esto se sitúa en la posibilidad de que los trabajadores, insatisfechos, se organicen de forma colectiva para reivindicar una mejora de sus 
condiciones de trabajo, lo que podría incluir estrategias que pongan en peligro el propio proceso de trabajo (ver Tassinari y Maccarrone, 2020).

En resumen, los repartidores están sujetos a un tipo de proceso que se puede describir como neotaylorista, por la necesidad que tiene de controlar y vigilar los tiempos y movimientos de los trabajadores para asegurarse la eficiencia productiva y que quizá resulta más llevadero, menos deshumanizado, por la interfaz rider-app que tiene claros componentes de gamificación. En relación con la lógica productivista, y en contradicción con lo anterior, los repartidores tienen una aparente o parcial autonomía para la fijación de sus objetivos, al tiempo que están sometidos a un control mercantil que les hace asumir en carne propia las fluctuaciones de la demanda, así como a un control clientelar en su relación con el resto de actores en el proceso. Por último, y no menos contradictorio, estas formas de control anteriores conviven con una lógica fluida, que basa su control en la incertidumbre, en la ausencia de certeza en las normas del proceso de trabajo, en los resultados a obtener a partir del esfuerzo realizado, o en la mera posibilidad de continuar en la organización.

\section{Gestión de la subjetividad}

En este apartado, vamos a analizar los esfuerzos ideológicos que desarrolla la plataforma para promover una(s) determinada(s) subjetividad(es) (Alvesson y Willmott, 2002). En puridad, no se trataría de un intento de gestión de la cultura organizacional (Willmott, 1993), en la medida en que no se dirige a miembros de la organización. Por esta circunstancia, y por el tipo de trabajo de que se trata, esta gestión de la subjetividad va a presentar similitudes, pero también diferencias, respecto de lo que se ha analizado en otros sectores diferentes. En esta gestión de la subjetividad, la interpelación se produce en unos aparentes términos de universalidad, que no haría distinciones por edad, género o etnia. Sin embargo, en el análisis veremos cómo algunas de las interpelaciones invisibilizan a las mujeres, dando una imagen del repartidor diverso en términos de edad, etnia y condición social, pero siempre masculino. Vamos a distinguir en el análisis cuatro diferentes identidades fomentadas por la organización.

\section{Rider como colaborador/socio}

La plataforma busca explícitamente que la relación con los repartidores se defina en términos de colaboración o asociación entre dos partes que libremente acuerdan trabajar conjuntamente: "los riders están en el centro...", "son los principales socios de la compañía". En ese sentido, se enfatiza que los riders no son personal, sino asociados autónomos que colaboran libremente en la realización de una actividad o reparto.

Esta interpelación como colaboradores se mantiene en los mensajes que reciben los riders de la empresa en los grupos de chats de la compañía. En estos, los repartidores reciben indicaciones que piden conectarse antes de la hora comprometida, o quedarse más allá del final de su turno, o bien a aplicar en los horarios de mayor demanda. Pero nunca en forma de órdenes, sino de educadas peticiones sin carácter obligatorio: “¿puedes revisar tu disponibilidad para la noche de hoy?", “¿podéis conectaros ya, que tenemos muchos pedidos esperando?" Eso sí, ya vimos cómo la compañía diseña mecanismos para "animar" a los repartidores a conectarse en esos momentos de mayor demanda, con penalizaciones para quienes no lo hacen suficientemente, en contra de la predicada libertad de los riders para fijar sus horarios. 
Sin embargo, la simetría se rompe cuando la organización busca fomentar en los riders, y no al revés, unos principios y valores, como seguridad y respeto hacia clientes y demás actores. Así, la empresa les dice a los riders: "la seguridad debe ser tu máxima prioridad" o "[debes] tratar con respeto a todas las personas con las que interactúes". Igualmente, la asimetría aparece en los principios que se autoimpone la organización, pues se compromete a proteger, apoyar e informar a los riders: "salvaguardar la seguridad de los riders es una prioridad", "[la empresa] informa con regularidad sobre la operativa de la plataforma". De esta forma, la asimetría en la colaboración entre plataforma y riders coloca a la primera en una posición como de "hermano mayor", preocupada por el bienestar de los segundos, sobre los que tendría una cierta obligación de protección ante cualquier eventualidad.

\section{Rider como mal colaborador/traidor}

Estas tensiones se manifiestan con especial rotundidad en una interpelación, relacionada con la primera, que sitúa a los repartidores como potenciales malos colaboradores, traidores a la confianza que debe presidir toda relación de colaboración: "Cualquier violación de lo dispuesto anteriormente puede suponer un incumplimiento grave de tu contrato de proveedor de servicios", "vigilamos activamente todas las actuaciones sospechosas y fraudulentas".

Este tipo de mensajes indicaría varias cuestiones. Primero, estos comportamientos se producen realmente, lo que muestra que los trabajadores tienen posibilidad de hacer trampas en su relación con el algoritmo y el procedimiento de trabajo. Al fin y al cabo, la compañía no tiene la posibilidad de ejercer una vigilancia directa hacia los trabajadores, sino mediada por las herramientas y dispositivos informáticos. Segundo, es otra muestra de la mencionada asimetría, pues esta capacidad sancionadora no es posible lógicamente en sentido inverso. Por último, esta indeterminación del trabajo, por utilizar el concepto de la Teoría del Proceso de Trabajo (Edwards, 1990), implica que es necesario algún tipo de autodisciplinamiento (Danaher, Schirato y Webb, 2000) del trabajador para aceptar las normas de la organización, aunque solo sea por propio interés y evitar las represalias. De ahí la apelación a la honestidad de los repartidores como valor que debe presidir su conducta: "Honestidad. Para ofrecer un servicio de calidad, los riders deben actuar de buena fe". Con esto la plataforma se convierte en una especie de vigilante inquisidor, preocupada por detectar las infracciones de los repartidores.

\section{Rider como persona}

Quizá menos habitual en los intentos de las organizaciones por regular la subjetividad de los trabajadores sea la interpelación en términos que remiten a la vida privada. Esto es absolutamente congruente con los valores de libertad y flexibilidad que fomenta la compañía en la relación con sus "colaboradores". Donde mejor se aprecian estos intentos de regulación de la subjetividad es en las historias personales de repartidores que algunas de estas plataformas ofrecen en sus páginas web, que se convierten en formas de identidad fomentadas por la organización. Es llamativo que todas las historias disponibles en la plataforma eran, en el momento de recogida de la información, de personas de diferente edad, etnia y condición social, pero siempre hombres.

En estas historias destaca cómo se enfatiza que el rider tiene su vida fuera, es decir, que aquello que más valora o a lo que dedica más tiempo no es a su trabajo como repartidor, sino a otras actividades, laborales o personales. Así, se muestra que no se pide un compromiso intenso con la empresa ni se espera una devoción por la compañía, como puede ser habitual en otros tipos de fomento de la cultura organizacional (Kunda, 1992), en 
línea con la flexibilidad y libertad que predica la plataforma. Así se plantea casi siempre la colaboración en términos de complemento de ingresos, indicativo de ese compromiso a tiempo parcial, que legitima unos ingresos limitados. En este sentido, se destaca la posibilidad del repartidor para elegir los horarios, sin que interfieran, aparentemente, con esa vida personal.

En esa vida fuera del reparto, los riders tienen familia y tienen hobbies, a los que dedican su atención y su tiempo. Con este tipo de descripciones se humaniza al repartidor, se le muestra como una persona particular y valorable, con lo que la compañía indica que se preocupa por tener en cuenta ese lado humano de sus "colaboradores", y también un cierto compromiso por no tratarles de forma indiferenciada, como un número más. Al tiempo, se presenta a la compañía como respetuosa de la vida privada de sus "colaboradores", incluso contribuyendo positivamente a la conciliación entre vida personal y vida privada. De hecho, en estas historias masculinas, la compatibilización del trabajo de repartidor con las obligaciones familiares nunca se presenta como un inconveniente. Es ciertamente sorprendente si consideramos que los horarios de reparto ocupan sobre todo las horas de comida y cena y especialmente en fin de semana, cuando las necesidades familiares pueden ser mayores. Pero, por otro lado, considerar la conciliación sería contradictorio con el omnipresente discurso organizacional de la flexibilidad (el trabajador elige sus horarios), que legitima buena parte de la organización del trabajo.

El repartidor también se muestra humanizado en su satisfacción con el trabajo de repartir, con su valoración del "buen ambiente" o "buen rollo" que preside la relación con la compañía y entre compañeros. Por tanto, como persona abierta, sociable, y con sentimientos que han de ser considerados. Las pequeñas críticas que aparecen, como referencias al estrés ("a veces puede ser muy estresante"), a los malos días que suceden a veces, etc., dan mayor credibilidad a la valoración positiva antes referida, al tiempo que contribuyen a humanizar aún más a los repartidores que narran sus historias.

Es llamativo también que muchos de estos repartidores aparecen embarcados en iniciativas emprendedoras propias, emergentes y todavía no consolidadas, por lo que no generan ingresos suficientes para permitir su autosuficiencia. Esto cumple al menos dos objetivos. Primero, da una imagen de los repartidores como personas activas, con intereses que sobrepasan su identidad como rider. Por tanto, refuerza la idea de que su vida está fuera de su relación con la plataforma. En segundo lugar, muestra a estos repartidores no como deseosos de convertirse en asalariados (o falsos autónomos), sino como emprendedores a los que la plataforma está ayudando indirectamente a desarrollar sus negocios, gracias a una flexibilidad de horarios que sería impensable en un trabajo asalariado convencional. Con ello se erige en un argumento indirecto de cara a las luchas por el reconocimiento legal de esta actividad laboral en los términos que establecen las plataformas.

De esto se deriva la apelación a un yo activo, casi hiperactivo, en todo caso productivo y eficiente, que es capaz de sacar partido económico a tiempos en muchos casos improductivos. Hay, pues, un llamado a "aprovechar el tiempo al máximo", como hacen estos repartidores, especialmente los emprendedores. Una de las historias de repartidores es especialmente llamativa a este respecto, pues a dos iniciativas empresariales "a las que dedica todo su tiempo", se unen sus horas de reparto con la plataforma y, además, aún le queda tiempo para actividades formativas. Independientemente de la precisión mayor o menor en la descripción del caso, lo que indican estos énfasis es un compromiso con una ideología del empresario de sí mismo, con individuos que muestren capacidades y disposiciones emprendedoras específicas (Du Gay, 1996).

Con esta interpelación la plataforma se coloca en una posición muy positiva, que le permite destacar su contribución al bienestar de su "colaboradores" y a la sociedad en general. 


\section{Rider como cliente}

La última interpelación que analizamos es la del repartidor como cliente. Esta perspectiva se hace posible por la existencia de un número importante de "colaboradores" que precisan las plataformas para atender su variable demanda. La clientelización de las relaciones de trabajo no es algo nuevo, en línea con la tendencia expuesta por Dubar (2002) a entender el trabajo como relación de servicio. En ese sentido, es frecuente actualmente la denominación de clientes internos a aquellos trabajadores de la propia compañía a los que un determinado departamento debe proveer de un determinado producto o servicio. Como señala el propio Dubar, cuando las relaciones se establecen como de servicio, la clave deja de estar en el producto o servicio producido y se sitúa más bien en la satisfacción de ese cliente interno.

En el caso de los repartidores asociados a plataformas, no se podría hablar en puridad de cliente interno, pero tampoco de cliente externo, sino que se configuraría una especie de estatus híbrido. Uno de los instrumentos más tradicionales relacionados con la clientelización de las relaciones de trabajo internas a la organización es el ofrecimiento de descuentos o beneficios no monetarios a los trabajadores. En ese sentido, la plataforma ajusta estos beneficios a las necesidades que entiende que tienen sus repartidores, como ayuda para la adquisición de vehículos, descuentos en comidas o cursos formativos. Esto no es muy distinto a los beneficios sociales o de otro tipo que tradicionalmente han ofrecido las empresas a sus trabajadores y que Revilla y Tovar (2011) relacionan con el control benevolente. La diferencia seguramente radique en que, en este caso, estos beneficios no tienen realmente un efecto notorio en la mejora de su situación precaria y, con ello, no tendrían el efecto motivador que podía tener el control benevolente en las empresas de régimen fordista. Por ello, parecen erigirse más bien en un elemento de fidelización a la plataforma ("engagement"), sobre todo respecto de la competencia con otras plataformas. De esta forma, se acerca a una de las estrategias de marketing más conocidas en la actualidad, la fidelización del cliente para retenerle y evitar su fuga a compañías de la competencia.

Por otro lado, la plataforma utiliza, de forma adaptada, otra herramienta que es propia de las relaciones de las empresas con los clientes, como son las encuestas que buscan conocer mejor sus características, necesidades o intereses, como forma de personalizar el servicio que ofrecen. En esta dirección, las encuestas de las plataformas hacia sus repartidores indagan sobre aspectos que indicarían dónde se encuentran las mayores dificultades percibidas por la compañía. Lógicamente, estos aspectos variarán a lo largo del tiempo en función de las transformaciones en la organización del trabajo (cambios en el algoritmo o en las políticas de la empresa). En la encuesta de la que disponemos, que data de 2017, la preocupación mayor parece situarse en el tipo de beneficios que puedan ser más atractivos para el repartidor (descuentos en productos, viajes, material de trabajo, actividades de ocio, vehículos o formación). Esto nos remite nuevamente a la necesidad de fidelizar al rider frente a la competencia.

La encuesta indaga también en la importancia que otorgan los repartidores a los distintos tipos de seguros (enfermedades, reclamaciones, daños personales) que la compañía podría proveer. De hecho, en la actualidad la compañía ofrece un seguro gratuito a todos sus riders, que cubre, durante el tiempo de trabajo, los daños que se produzcan en caso de accidente, así como su eventual responsabilidad civil. No así en caso de enfermedad común. Esto va en la línea de su proclamada preocupación por la seguridad, que señalamos más arriba, y atiende uno de los problemas más señalados en las críticas a esta forma de trabajo, precisamente por su carácter no asalariado: la desprotección del repartidor frente a cualquier accidente que pueda suceder en su movilidad por el peligroso tráfico urbano. Refuerza con ello también la ya mencionada imagen de "hermano mayor". 
Igualmente, la compañía dedica atención en la encuesta a la importancia que otorgan los repartidores a distintas cuestiones relacionadas con los procedimientos de trabajo: formas de organizar los tiempos de trabajo, modalidades distintas de establecer el pago por reparto, comunicación entre compañía y riders, o relaciones entre estos. Con ello, la plataforma permite una limitada forma de participación entre los repartidores, que refuerza esa interpelación en términos de clientes o usuarios y coloca a la compañía como proveedora benevolente.

En resumen, hemos visto cómo las interpelaciones de la plataforma a la subjetividad de sus repartidores se dirigen a reforzar su estatus de trabajadores autónomos, no asalariados, sino colaboradores, clientes o simplemente personas (hombres) con una vida personal propia que no interfiere en su trabajo. En este sentido, es esta una forma de gestión de la subjetividad bastante distinta a las que se pueden ver en organizaciones innovadoras, que enfatizan el compromiso intenso con el proyecto colectivo, la implicación subjetiva con la compañía o la pertenencia a un equipo o a una familia. Como de hecho sucede en el caso de los trabajadores que la plataforma reconoce como propios.

Por supuesto, estos intentos de la plataforma en la gestión de la subjetividad no están exentos de tensiones o contradicciones. La relación de colaboración muestra una asimetría evidente entre las partes, que sitúa a la compañía entre un "hermano mayor" protector y proveedor y un vigilante inquisidor de las infracciones de sus repartidores. Al tiempo, la interpelación en términos de respeto a la vida privada individual se enfrenta a unos procedimientos de trabajo altamente neotayloristas, que necesitan de un elevado control de los comportamientos de los riders, contradiciendo la tan destacada flexibilidad y libertad de la que disfrutarían.

\section{CONCLUSIONES}

Mediante el análisis realizado hemos podido mostrar cómo la situación laboral de los repartidores en las plataformas de comida a domicilio supone un cambio significativo en las formas en que se desarrolla este proceso y en sus lógicas organizacionales. Este es precisamente el factor disruptivo en el ámbito laboral que estas plataformas reclaman para sí.

Sin embargo, este cambio tiene que ver con la agregación y solapamiento de distintas lógicas disciplinarias previamente existentes, algunas con ciertas modificaciones, fruto de las innovaciones que aportan estas formas de organización del trabajo, que confluyen y se confunden en la posición laboral de repartidor. Con ello, se generan tensiones y contradicciones inherentes a estos sistemas de control organizacional, que, si bien parecen estar dirigidos a garantizar su buen funcionamiento, propiciando así un aumento en la productividad de la actividad, convierten a esta posición laboral en dificilmente vivible.

Es llamativo, en ese sentido, cómo el proceso laboral incorpora una nueva forma de medición de espacios y tiempos y de vigilancia al margen de espacios cerrados de trabajo (neotaylorismo ubicuo), si bien este proceso puede ser presentado a la vez $-y$ contradictoriamente- bajo un carácter más humanizado, típico del entorno de juego que representa el concepto de gamificación. Al tiempo, se recurre a lógicas de control basadas en la incertidumbre, más típicas del modo de regulación postfordista y su flexibilidad fundacional.

Curiosamente, son los desarrollos tecnológicos innovadores basados en algoritmos los que están haciendo posible ambas cuestiones a la vez. Por un lado, permiten, y precisan, un control absoluto sobre el trabajador, en una reedición del trabajador tayloriano como "apéndice de la máquina". Por otro, la opacidad y dificultad de comprensión del funcionamiento del algoritmo produce una suerte de mistificación de la tecnología que legitima casi cualquiera de sus desarrollos y de los efectos sobre el proceso de trabajo que 
pueda producir. Todo ello permite a la compañía externalizar las variaciones de la demanda en su mercado propio, que acaba recayendo en los repartidores, en una nueva forma de control mercantil.

En este sentido, los algoritmos no sólo servirían para la gestión optimizada de masivas cantidades de datos, sino también, y probablemente con una significatividad mayor, para la gestión de la subjetividad que implican los procesos laborales. Así, los cambios que han propiciado estas nuevas formas de control organizacional no se darían tanto en la materialidad de la actividad, como, precisamente, en la gestión de la subjetividad que implica el ámbito de trabajo que hemos analizado. De hecho, es posible pensar en menores cuotas de control y vigilancia en formas previas de reparto de comida a domicilio. Pero entendemos que el desarrollo de estas nuevas formas de disciplinamiento, contradictorias como son entre sí, hacen más necesarios precisamente esos esfuerzos de la organización por gestionar la subjetividad de los repartidores. La interpelación a estos en cuanto trabajadores autónomos, colaboradores, personas (hombres) con vida propia cumple la función de legitimar la falta de compromiso de la compañía hacia sus repartidores, legitimando de paso que los ajustes en las fluctuaciones de la demanda recaigan sobre ellos. Lo interesante también, desde este punto de vista, es cómo la organización no hace descansar en la organización del proceso de trabajo, y por tanto en los algoritmos, esta gestión de la subjetividad, sino que arbitra mecanismos propios para fomentar estos discursos sobre la relación entre trabajador y compañía.

Por supuesto, las contradicciones son también notorias entre algunas lógicas disciplinarias y este control de la subjetividad. El discurso de la autonomía que enfatiza la plataforma chocaría con el alto nivel de formalización implícito en el proceso de trabajo y con esa imagen de "hermano mayor" implícita también en el discurso de la plataforma. Un "hermano mayor" que, sin el compromiso y protección de otras formas laborales no autónomas, deja a los riders expuestos al mercado, según ha mostrado el análisis de la presencia de las lógicas disciplinarias productivistas y mercantiles. Protege y provee porque necesita vigilar, y por ello los repartidores deben utilizar los recursos tecnológicos que este hermano benefactor pone a su disposición, pero, en su ensamblaje con el discurso del respeto a la vida privada y a la autonomía de los repartidores, se desentiende de las consecuencias que el proceso productivo pueda significar para los repartidores.

Por tanto, en torno a la posición laboral de los riders, se genera un contexto material y discursivo en el que las lógicas de la precariedad y el emprendimiento, entendidas como instrumentos disciplinarios, dibujan una situación tan altamente contradictoria que parece imposible, o al menos bastante dificil, de vivir, lo que les convierte en trabajadores incómodos (uneasy riders).

\section{REFERENCIAS}

Alonso, L.E. (2017). Consumo colaborativo: las razones de un debate. Revista Española de Sociología, 26(1), 87-95.

Alonso, L.E. y Fernández, C.J. (2009). Uso del trabajo y formas de gobernabilidad: la precariedad como herramienta disciplinaria. En E. Crespo, C. Prieto y A. Serrano (eds.), Trabajo, subjetividad y ciudadanía. Paradojas del empleo en una sociedad en transformación (pp. 229-258). Madrid: CIS-UCM.

Alvesson, M. y Willmott, H. (2002). Identity regulation as organizational control: producing the appropriate individual. Journal of Management Studies, 39(5), 619-644.

Anderson, L. (2006). Analytic autoethnography. Journal of Contemporary Ethnography, 35, 373-395. 
Barker, J.R. (1993). Tightening the Iron Cage: Concertive Control in Self-Managed Teams. Administrative Science Quarterly, 38(3), 408-437.

Berger, P.L. y Luckman, T. (1986). La construcción social de la realidad. Argentina: Amorrortu Editores.

Bodo, B., Helberger, N., Irion, K., Zuiderveen Borgesius, F., Moller, J., van de Velde, B., Bol, N., van Es, B. y de Vreese, C. (2017). Tackling the Algorithmic Control Crisis. The Technical, Legal, and Ethical Challenges of Research into Algorithmic Agents. Yale Journal of Law and Technology, 19(1), 133-180.

Boltanski, L. y Chiapello, E. (1999). El nuevo espíritu del capitalismo. Madrid: Akal.

Bourdieu, P. (2006). Autoanálisis de un sociólogo. Barcelona: Anagrama.

Burawoy, M. (1979). Manufacturing consent. Berkeley: University of California Press.

Cameron, L. (2020). The Rise of Algorithmic Work: Implications for Organizational Control and Worker Autonomy (PhD Dissertation). University of Michigan.

Cant, C. (2020). Riding for Deliveroo. Resistance in the New Economy. Cambridge: Polity Press.

Cherry, M.A. (2016). Beyond misclassification: The digital transformation of work. Comparative Labor Law y Policy Journal, 37(3), 577-602.

Danaher, G., Schirato, T. y Webb, J. (2000). Understanding Foucault. Londres: Sage.

De Stefano, V. (2016). The rise of the "just-in-time workforce": on-demand work, crowdwork and labour protection in the "gig-economy". Geneva: International Labour Office -ILO.

Denzin, N.K. (1997). Interpretive Ethnography: ethnographic practices of the 21st century. Londres: Sage Publications.

Drucker, P.F. (1979). Managing for Results. Londres: Pan Books.

Du Gay, P. (1996). Organización de la identidad: gobierno empresarial y gestión pública. En S. Hall y P. Du Gay (comps.), Cuestiones de identidad cultural (pp. 251-281). Buenos Aires: Amorrortu.

Dubar, C. (2002). La crisis de las identidades: la interpretación de una mutación. Barcelona: Bellaterra.

Edwards, P.K. (1990). Understanding conflict in the labour process: The logic and autonomy of struggle. En D. Knights y H. Willmott (eds.), Labour Process Theory (pp. 125-152). Basingstoke: Palgrave Macmillan.

Ellis, C., Adams, T. y Bochner, A. (2011). Autoethnography: An Overview. Historical Social Research / Historische Sozialforschung, 36(4 (138)), 273-290. http://www.jstor.org/ stable/23032294

Fleming, P. y Sewell, G. (2002). Looking for the Good Soldier, Švejk: Alternative Modalities of Resistance in the Contemporary Workplace. Sociology, 36(4), 857-873.

Frenkel, S., Korczynski, M., Donoghue, L. y Shire, K. (1995). Re-constituting work: Trends towards knowledge work and info-normative control. Work, Employment and Society, 9(4), 773-796.

Hammersley, M. y Atkinson, P. (1994). Etnografía. Métodos de investigación. Barcelona: Paidós. 
Huber, C. y Scheytt, T. (2018). Pitfalls of algorithmic control and their implications for support systems Algorithmic control as a threat to accountability. En R. Weidner y A. Karafillidis, Technische Unterstützungssysteme, die die Menschen wirklich wollen (pp. 205-212). Hamburg: Helmut-Schmidt-Universität.

Huws, U. (2014). Labour in the Global Digital Economy. The Cybertariat comes of age. New York: Monthly Review Press.

Huws, U., Spencer, N., Syrdal, D. y Holts, K. (2017). Work in the European Gig Economy. Brussels: Foundation for European Progressive Studies -FEPS, UNI Europa, University of Hertfordshire.

Kunda, G. (1992). Engineering Culture. Control and Commitment in a High-Tech Corporation. Philadelphia: Temple University Press.

Lee, M.K., Kusbit, D., Metsky, E. y Dabbish, L. (2015). Working with machines: The impact of algorithmic and data-driven management on human workers. En Proceedings of the 33rd Annual ACM Conference Conference Extended Abstracts on Human Factors in Computing Systems (pp. 1603-1612). New York: Association for Computing Machinery.

Pesole, A., Urzí Brancati, M.C., Fernández-Macías, E., Biagi, F. y González Vázquez, I. (2018). Platforms Workers in Europe. Evidence from the COLLEEM Survey. Luxembourg: JRC Science Hub (European Union).

Piñeiro, C., Surinach, R. y Casadevante, J.L. (2017). Entre el mercado y la cooperación social. Luces y sombras de las prácticas de consumo colaborativo. Revista Española de Sociología, 26(1), 97-108.

Revilla, J.C. y Tovar, F.J. (2011). El control organizacional en el siglo XXI: en busca del trabajador autodisciplinado. REIS, 135, 47-68.

Schildt, H. (2017). Big data and organizational design - the brave new world of algorithmic management and computer augmented transparency. Innovation, 19(1), 23-30.

Sewell, G. y Wilkinson, B. (1992). 'Someone to watch over me': Surveillance, Discipline and the Just-in-Time Labour Process. Sociology, 26(2), 271-289.

Tassinari, A. y Maccarrone, V. (2020). Riders on the Storm: Workplace Solidarity among Gig Economy Couriers in Italy and the UK. Work, Employment and Society, 34(1) 35-54.

Valenduc, G. y Vendramin, P. (2017). Digitalisation, between disruption and evolution. Transfer, 23(2), 121-134.

Veen, A., Barratt, T. y Goods, C. (2020). Platform-capital's 'app-etite' for control: a labour process analysis of food-delivery work in Australia. Work, Employment and Society, 34(3), 388-406.

Willmott, H. (1993). 'Strength is ignorance; slavery is freedom': managing culture in modern organizations. Journal of Management Studies, 30(4), 515-552. 


\section{NOTAS BIOGRÁFICAS}

Juan Carlos Revilla es profesor titular de Psicología Social y subdirector del departamento de Antropología Social y Psicología Social de la UCM en la Fac. de CC. Políticas y Sociología. Sus líneas de investigación son los estudios críticos del trabajo y las organizaciones, la identidad social, los estudios de juventud y la violencia social. Ha participado en numerosos proyectos de investigación, destacando la coordinación nacional de dos proyectos europeos del 70 Programa Marco (SPHERE y RESCuE). Es miembro del Instituto de Investigación TRANSOC (UCM).

Víctor Blázquez es doctor en Sociología y profesor asociado en el departamento de Historia, Teorías y Geografia Políticas de la UCM, en la Facultad de CC. Políticas y Sociología. También ha sido profesor asociado en el departamento de Pedagogía de la UAM, en la Facultad de Educación y Formación del Profesorado. Sus líneas de investigación han ido desde la cooperación al desarrollo a los procesos laborales o las TIC. Amplia trayectoria profesional en sector logístico y consultoría. 